Cite this: J. Mater. Chem. C, 2013, 1, 7758

Received 3rd June 2013

Accepted 9th October 2013

DOI: $10.1039 / \mathrm{c} 3 \mathrm{tc} 31055 \mathrm{~h}$

www.rsc.org/MaterialsC

\section{Enhanced interfacial rigidity of 1D thermoset nanostructures by interface-induced liquid crystallinity $\dagger$}

\author{
Hatice Duran, ${ }^{\text {a }}$ Basit Yameen, ${ }^{\mathrm{b}}$ Markus Geuss,,$_{+}^{\mathrm{c}}$ Micheal Kappl, ${ }^{\mathrm{d}}$ Martin Steinhart ${ }^{\mathrm{e}}$ \\ and Wolfgang Knoll ${ }^{f}$
}

Polycyanurate networks (PCNs), which form random networks in the bulk, are representative of an important class of thermosetting materials. We show that free surfaces of PCNs exhibit rigidity enhanced by one order of magnitude (quantified by Young's modulus) if they are initially synthesized in the presence of hard confining interfaces, such as the pore walls of nanoporous anodic aluminum oxide (AAO). Using self-ordered AAO, which contains arrays of aligned cylindrical nanopores uniform in length and diameter as an inorganic model matrix, we could evidence interface-induced liquidcrystalline ordering of the liquid cyanate ester monomers (CEMs) at the pore walls. The interfacial ordering of the CEMs, which is conserved upon curing, is most likely the origin of enhanced rigidity of the free PCN surfaces after release of the one-dimensional PCN nanostructures from AAO. The results presented here should be of considerable relevance for the processing of industrially relevant thermosets, for the understanding of polymer/solid interfaces, for the design of advanced nanocomposites for applications in aviation and high-speed electronics, and for the design of mechanical hybrid nanostructures for advanced biomimetic adhesive systems.

\section{Introduction}

Polycyanurate thermosets are made from cyanate esters (CEs), a new generation of thermosetting resins. ${ }^{1,2}$ The polycyanurates derived from the CEs exhibit attractive dielectric properties, high thermal stability and low shrinkage, the single component curing releases almost no volatile components. ${ }^{3-6}$ CEs undergo thermally induced cyclotrimerization reactions resulting in the formation of three-dimensional polycyanurate networks (PCNs) which have much higher thermal resistance than epoxy resins. ${ }^{7}$ Typical fabrication processes involving CEs, such as resin

${ }^{a}$ TOBB University of Economics and Technology, Department of Materials Science and Nanotechnology Engineering, Söğütözü Cad. 43, 06560 Ankara, Turkey. E-mail: hduran@etu.edu.tr; Fax: +90 312292 4372; Tel: +90 3122924339

${ }^{b}$ Lahore University of Management Sciences (LUMS), School of Science and Engineering (SSE), Dept. of Chemistry, 54792 Lahore, Pakistan. E-mail: basit.yameen@lums. edu.pk

${ }^{c}$ Max Planck Institute of Microstructure Physics, Weinberg 2, D-06120 Halle, Germany ${ }^{d}$ Max Planck Institute for Polymer Research, Ackermannweg 10, D-55128 Mainz, Germany.E-mail: kappl@mpip-mainz.mpg.de

${ }^{e}$ Universität Osnabrück, Institut für Chemie neuer Materialien, D-49069 Osnabrück, Germany.E-mail: martin.steinhart@uos.de

${ }^{f}$ AIT Austrian Institute of Technology, Donau-City-Straße 1, 1220 Vienna, Austria. E-mail: Wolfgang.Knoll@ait.ac.at

$\dagger$ Electronic supplementary information (ESI) available: DSC, POM and WAXS characterization and $I_{\text {trans }}$ derivation. See DOI: 10.1039/c3tc31055h

\$ Current address: University of Applied Science of Western Switzerland, College of Engineering and Architecture, Bd de Pérolles 80, P. O. Box 32, CH-1705 Fribourg, Switzerland. E-mail: markus.geuss@hefr.ch transfer molding, ${ }^{8}$ nanostructure casting ${ }^{1}$ and prepregging ${ }^{9}$ onto fibers, inevitably involve direct interactions between the CEs and solid surfaces. Moreover, interfacial interactions between inorganic filler particles and the CEs will influence the properties of the derived composites. Hence, understanding the influence of hard confining interfaces on the physical and mechanical properties of thermosets is central to the rational design and performance optimization of thermoset resins. Selfordered anodic aluminium oxide (AAO) containing arrays of aligned cylindrical nanopores with narrow size distribution (Fig. S1 $\dagger$ ) is an ideal model matrix for the investigation of the behaviour of thermosets at hard inorganic surfaces. AAO combines large internal interface area and, therefore, high interface volume fractions of infiltrated polymeric materials, with well-defined anisotropy. Hence, anisotropic features in interfaces at the pore walls can be probed, e.g., by X-ray texture analysis. ${ }^{10,11}$

Surface-induced order inside nanoporous templates at temperatures where bulk liquid phases are stable was previously reported for $n$-alkanes,,$^{12}$ non-entangled to weakly entangled polymers ${ }^{13}$ and liquid crystals in both experimental $^{14-19}$ and theoretical ${ }^{20}$ studies. For example, in nanoporous matrices nematic liquid crystals form paranematic phases characterized by surface-induced ordering in the immediate vicinity of the solid/liquid interface. For longchain $n$-alkanes rectified monolayers at pore walls were reported even at temperatures above the surface-freezing temperature. ${ }^{12,21}$ 
We developed a cyanate ester monomer (CEM) based on oligomeric aryl ether derivatives of bisphenol AF which differs from commercially available and structurally related CEMs in that the oligomer backbone is functionalized with an additional cyanate group. CEM was derived from the phenolic precursors of oligomeric mixtures of linear polyethers bearing pendent phenolic groups as well as phenolic end groups. Commonly, phenols, such as the CEM precursors (phenolic oligomeric mixtures of aromatic polyethers), are solids because of the presence of hydrogen bonds. However, substituting the phenolic groups by cyanate groups results in the deprivation of hydrogen bonds and consequently in lower melting points. Like its phenolic precursors, CEM consists of a mixture of oligomers with different molecular weights. Missing repeat units of shorter CEM molecules can be considered as defects impeding defect-free crystallization of longer CEM. Thus, CEM is liquid at room temperature and exhibits pronounced stability against oxidation and moisture. ${ }^{1}$

Taking advantage of the liquid physical state of the employed CEM, we infiltrated CEM into self-ordered AAO (pore diameter $380 \mathrm{~nm}$; pore depth $100 \mu \mathrm{m}){ }^{22}$ Using AAO/PCN hybrids as a model system, we show that PCNs obtained from CEM show liquid-crystalline near-ordering induced by hard inorganic surfaces. Free PCN surfaces with liquid-crystalline near-ordering exhibit, as compared to PCN surfaces cured in the absence of hard counterpart surfaces, significantly enhanced rigidity.

\section{Experimental details}

\section{Materials and methods}

The synthesis and properties of CEM were described elsewhere. ${ }^{1}$ CEM - an oligomeric aryl ether derivative of bisphenol A with pendant and terminal cyanate groups - is a viscous liquid (the viscosity at $25^{\circ} \mathrm{C}$ is $3 \mathrm{~Pa} \mathrm{~s}$ ). The glass transition temperature $T_{\mathrm{g}}$ of CEM is about $-14.5{ }^{\circ} \mathrm{C}$, as determined by differential scanning calorimetry (see below). Field desorption mass spectroscopy and gel permeation chromatography analyses revealed that CEM consists of a mixture of monomers (45\%) and dimers $(55 \%)$ with an average molecular weight of $1190 \mathrm{~g} \mathrm{~mol}^{-1}$.

\section{Preparation of bulk PCN thermosets}

CEM was degassed at $80{ }^{\circ} \mathrm{C}$ for $1 \mathrm{~h}$. To prepare thin bulk PCNs, neat CEM was cured in a Teflon mold placed in a tube furnace under argon by applying the following temperature program: $180{ }^{\circ} \mathrm{C} / 2 \mathrm{~h} \rightarrow 260{ }^{\circ} \mathrm{C} / 8 \mathrm{~h} \rightarrow 290{ }^{\circ} \mathrm{C} / 1 \mathrm{~h} \rightarrow$ cooling to room temperature. For WAXS measurements, the obtained PCNs were cut to $150 \mu \mathrm{m}$ thick films with a sharp blade.

\section{Preparation of CEM nanotubes and CEM nanorods}

CEM was deposited on the AAO membranes by spin coating (3000 rpm, $2 \mathrm{~min}$ ) at room temperature. Then, the CEM-coated AAO membranes were heated to $80{ }^{\circ} \mathrm{C}$ for obtaining CEM nanotubes and to $120{ }^{\circ} \mathrm{C}$ for obtaining CEM nanorods for $12 \mathrm{~h}$ under vacuum. Then, the CEM-infiltrated AAO samples were quenched with liquid nitrogen in order to solidify the infiltrate, and residual CEM was removed with sharp razor blades and soft polishing paper (Buehler Microcloth).

\section{Preparation of PCN nanotubes (PCTs) and solid PCN nanorods (PCRs)}

Prior to the infiltration with CEM, empty AAO membranes were weighed using a microbalance (Mettler-Toledo, MX5) with an accuracy of $1 \pm 0.8 \mu \mathrm{g}$ at least three times. CEM was deposited on the AAO membranes by spin coating (3000 rpm, $2 \mathrm{~min}$ ) at room temperature. Then, the CEM-coated AAO membranes were heated to either $80^{\circ} \mathrm{C}$ or $120^{\circ} \mathrm{C}$ for $12 \mathrm{~h}$ under vacuum. For curing, CEM-infiltrated AAO was annealed at $260{ }^{\circ} \mathrm{C}$ for $8 \mathrm{~h}$ and at $290{ }^{\circ} \mathrm{C}$ for $1 \mathrm{~h}$ in a tube furnace purged with $\mathrm{N}_{2}$. Then, the residual PCN was removed from the AAO surfaces with sharp razor blades and soft polishing paper (Buehler Microcloth). Finally, the PCN-containing AAO membranes were again weighed. The mass difference to empty AAO was assumed to correspond to the mass of the PCN located in the AAO membranes. Depending on the applied temperature, either PCTs or PCRs formed in the AAO pores (see below). Released PCTs and PCRs were obtained by selectively dissolving the AAO in aqueous $10 \mathrm{wt} \% \mathrm{NaOH}$ solution at $25{ }^{\circ} \mathrm{C}$ for $6-8 \mathrm{~h}$. The suspended PCTs and PCRs were centrifuged $(20000 \mathrm{rpm}$, $15 \mathrm{~min}$ ) and the supernatant liquid was removed. The released PCTs and PCNs were redispersed in deionized water and again centrifuged. This procedure was repeated until the supernatant liquid became neutral.

\section{Scanning electron microscopy (SEM)}

Scanning electron microscopy (SEM) investigations were performed using a LEO Gemini 1530 SEM operated at acceleration voltages ranging from 0.75 to $6 \mathrm{kV}$.

\section{Measurements of Young's modulus of individual PCTs}

Young's modulus of individual PCTs was measured by AFM (Veeco Dimension 3100) force spectroscopy with cantilevers (Olympus OMCL-AC 240 TS) having a spring constant of $2.0 \pm$ $0.2 \mathrm{~N} \mathrm{~m}^{-1}$, as determined by the thermal noise method. ${ }^{23}$ A drop of an aqueous suspension containing $1 \mathrm{wt} \%$ PCTs was deposited on a clean glass substrate. After evaporation of the water at room temperature, tapping mode AFM imaging was used to locate isolated nanotubes lying flat on the glass surface and to position the AFM tip just above the ridge of the probed PCT by successive reduction of the scan size. Typically, 100 forcedistance curves were taken on a single spot on each probed PCT. The maximum deflection of the cantilever was kept below $20 \mathrm{~nm}$ (corresponding to a maximum force below $40 \mathrm{nN}$ and an elastic tube deformation of a few $\mathrm{nm}$ ). Details of the method are described elsewhere. ${ }^{24}$ No indication of indentation of the PCT surface could be found by AFM imaging of the same spot after the force experiments, unless cantilever deflections in excess of $100 \mathrm{~nm}$ were applied. Before and after each series of force measurements on a PCT, reference force curves were taken on the glass substrate. Assuming that the PCT walls are very thin $(0.002<t / R<0.1 ; R$ is the PCT radius, $t$ the tube wall thickness), Young's modulus $E$ of a PCT can be calculated as follows: 


$$
E \approx \frac{k_{\mathrm{t}} R^{3 / 2}}{1.18 t^{5 / 2}}
$$

where $k_{\mathrm{t}}$ is the effective spring constant of a PCT that is placed on a hard substrate and subjected to a point force load. ${ }^{25}$ Since the prefactor of 1.18 in equation is almost constant up to $t / R$ values of 0.1 and $t / R$ amounted to $\sim 0.13$ in our measurements, eqn (1) should be valid.

\section{Polarizing optical microscopy (POM)}

Birefringence indicative of anisotropic ordering in the probed samples was measured by POM using a Nikon Optipot 2-POL microscope with a filtered halogen light source $(12 \mathrm{~V}, 100 \mathrm{~W})$. Images were captured using the video image capture software Snappy Video Snapshot, version 2.0. Angle-dependent brightness profiles were obtained by using the image analysis software Image J. The average birefringence at $45^{\circ}$ was calculated using a Michel-Lévy interference chart supplied by Nikon MicroscopyU. A Michel-Lévy graph plots retardation on the abscissa and specimen thickness on the ordinate. Birefringence was determined by comparing the highest-order interference color detected in the course of a measurement to those contained on the chart.

\section{Differential scanning calorimetry (DSC)}

DSC measurements were carried out with a Mettler Toledo Star DSC. The samples were first heated from room temperature to $350{ }^{\circ} \mathrm{C}$ at a rate of $10{ }^{\circ} \mathrm{C} \mathrm{m^{-1 }}$ and then cooled to $-100{ }^{\circ} \mathrm{C}$ at a rate of $-10{ }^{\circ} \mathrm{C} \mathrm{min}^{-1}$. For measurements on bulk PCN as well as on released PCTs and PCNs, 5-10 $\mathrm{mg}$ sample material was placed in hermetically sealed pans. For measurements on PCN located in AAO, the mass fraction of PCN was estimated from the mass difference between PCN-containing and empty AAO. Prior to the DSC measurements, the Al substrate to which the AAO layer had been attached was etched away using a mixture of $\mathrm{HCl}, \mathrm{CuCl}_{2}$, and $\mathrm{H}_{2} \mathrm{O}$.

\section{Wide-angle X-ray scattering (WAXS)}

$\mathrm{X}$-ray diffraction measurements were carried out on a Philips X'pert MRD diffractometer operated with $\mathrm{Cu} \mathrm{K} \alpha$ radiation $(\lambda=$ $0.154 \mathrm{~nm}$ ) at $40 \mathrm{kV}$ and $40 \mathrm{~mA}$. During any X-ray measurement, CEM nanorods, CEM nanotubes, PCRs and PCTs were still located in the AAO membranes. For $\theta / 2 \theta$ scans, the AAO membranes were mounted on a Eulerian cradle in such a way that the AAO pore axes were oriented parallel to the plane of incident beam and detector (incident beam and AAO surface enclosed the scattering angle $\theta$ ). The samples were tilted about the $\theta$ axis by an angle $\theta$, while the detector was rotated about the same axis by an angle $2 \theta$. The $\theta$ axis was oriented normal to the AAO pore axes and to the scattering plane defined by the incident beam and the position of the detector. Thus, in $\theta / 2 \theta$ scans only crystals having the corresponding lattice planes oriented parallel to the AAO surface (normal to the AAO pore axes) contributed to the detected intensity of specific reflections.

Schulz scans ${ }^{\mathbf{1 0 , 1 1 , 2 6}}$ yield orientation distributions of specific sets of lattice planes relative to the AAO surface or, equivalently, orientation distributions of the corresponding reciprocal lattice vectors relative to the AAO pore axes. Schulz scans were measured for fixed scattering angles $\theta$. The AAO membranes were tilted by an angle $\Psi$ about the $\Psi$ axis lying in the scattering plane but being oriented perpendicularly with respect to the $\theta$ axis. As a result, scattering intensity profiles $I(\Psi)$ along Debye rings belonging to the fixed scattering angle $\theta$ were obtained (a Debye ring is the circle of intersection of the Ewald sphere and a sphere about the origin of the reciprocal space with a radius corresponding to the length of the scattering vector belonging to $\theta$ ). The apparent $I(\Psi)$ values sharply decrease for high $\Psi$ angles $>\sim 70^{\circ}$ because of defocusing effects. ${ }^{27}$ Prior to any measurement, residual material on the surface of the AAO membranes was removed with sharp blades and polishing paper. All measurements were performed at room temperature.

\section{Results and discussion}

After the spin coating step, a liquid CEM reservoir covers the AAO membranes. Organic materials with low surface energies, such as CEM, commonly spread on inorganic surfaces with high surface energies such as the AAO pore walls. ${ }^{28}$ Therefore, covering AAO pore walls with CEM reduces the surface energy of the entire system. While in equilibrium the AAO pores will be completely filled with CEM (so that any AAO/air and CEM/air interface vanishes), infiltration kinetics is complex, and instable intermediate states might be frozen. Two limiting cases for the infiltration of spreading organic liquids into cylindrical pores have been identified: precursor wetting and capillary wetting. In the course of capillary wetting, liquid threads spanning across the entire cross section of the pore preceded by a meniscus move into the pore. Capillary wetting only dominates if the infiltrated liquid is highly viscous and has predominantly been observed for highly entangled polymer melts $^{29}$ and microphase-separated block copolymers. ${ }^{30}$

Precursor wetting, which commonly occurs if the infiltrated liquid has low or medium viscosity, involves rapid formation of microscopic to mesoscopic precursor films on the pore walls. ${ }^{31,32}$ If the pore diameter is large enough (typically above a few tens of $\mathrm{nm}$ ), the annular precursor films surround cylindrical hollow volumes. Complete filling of the pore volumes occurs via the "snap-off" mechanism or by gradual thickening of the precursor film starting from the pore mouths. ${ }^{33}$ The "snap-off" mechanism involves the development of menisci at instabilities of the annular precursor films. As more infiltrated liquid flows into the pores, the liquid flows into the menisci. As a result, the interfaces of the menisci move in the opposite direction until the pores are completely filled with liquid. ${ }^{34,35}$ When CEM is infiltrated into AAO at $80{ }^{\circ} \mathrm{C}$, CEM develops annular precursor films on the AAO pore walls. However, at this temperature, CEM is viscous enough to slow down further filling of the AAO pores by any of the mechanisms mentioned above. Therefore, annular CEM precursor films persist on the AAO pore walls that can be converted into PCTs by curing.

At $120{ }^{\circ} \mathrm{C}$, the viscosity of CEM is lower than at $80{ }^{\circ} \mathrm{C}$. Thus, CEM molecules do not only form annular precursor films on the AAO pore walls. Owing to the higher mobility of the CEM 
molecules at $120{ }^{\circ} \mathrm{C}$, CEM fills the entire pore volume by the "snap-off" mechanism or by precursor film thickening much faster than at $80{ }^{\circ} \mathrm{C}$. Curing then yields solid PCRs. Selective etching of the AAO yields released PCTs obtained by CEM infiltration at $80{ }^{\circ} \mathrm{C}$ (Fig. 1a) or PCRs obtained by CEM infiltration at $120^{\circ} \mathrm{C}$ (Fig. 1b). Both PCTs and PCRs had a length of $100 \mu \mathrm{m}$ and a diameter of $380 \mathrm{~nm}$; i.e., they were faithful negative replicas of the AAO nanopores.

Atomic force microscopy measurements of the mechanical properties of single PCTs after their release from the AAO membranes were carried out as described above. Fig. 2a shows an AFM image of a single PCT on a flat alumina substrate, while Fig. 2b shows a scanning electron microscopy (SEM) image of a single PCT $380 \mathrm{~nm}$ in diameter having a wall thickness of $20 \mathrm{~nm}$. To obtain the Young's modulus from eqn (1), the effective spring constant $k_{\mathrm{t}}$ of each PCT was calculated from the slopes of the force curves (Fig. 2c) in the region of contact between tip and PCT, as determined by linear fits:

$$
k_{\mathrm{t}}=\frac{a_{\mathrm{t}}}{a_{\mathrm{ref}}-a_{\mathrm{t}}} \cdot k
$$

In eqn (2) $a_{\mathrm{t}}$ is the slope of the force curves taken on PCTs, $a_{\mathrm{ref}}$ is the slope of the reference force curves taken on the glass

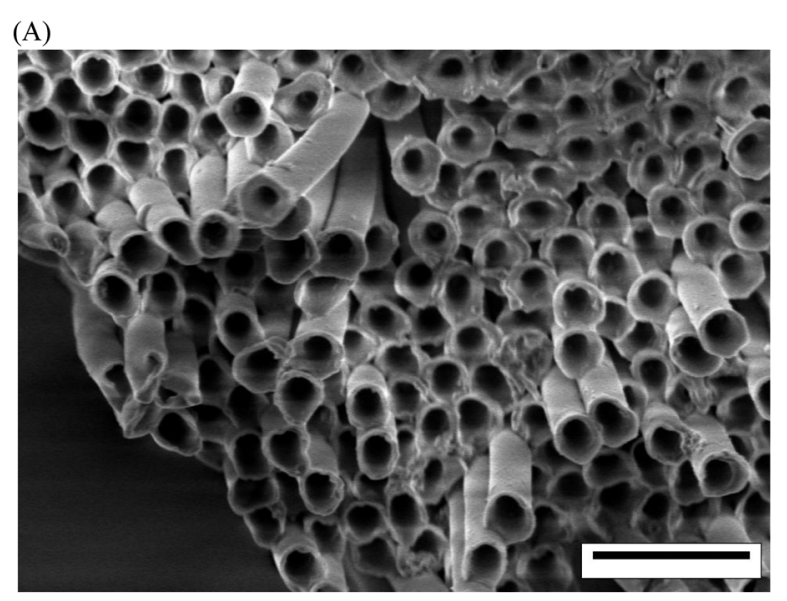

(B)

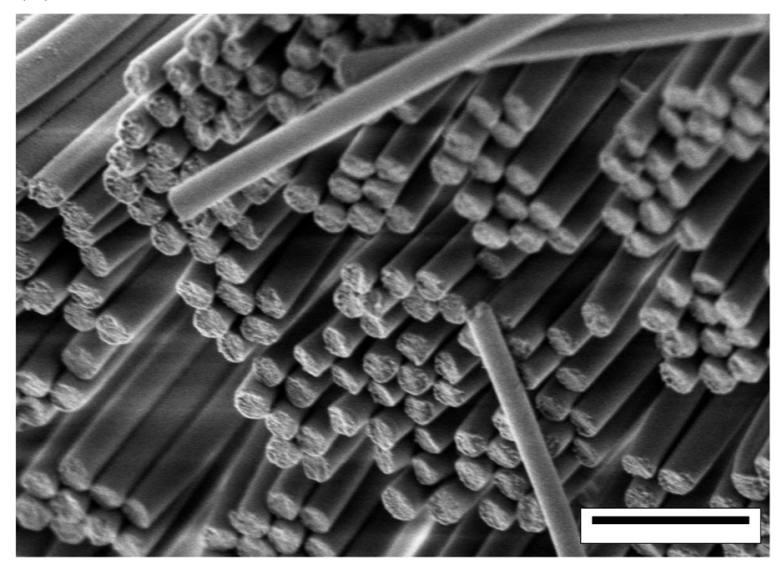

Fig. 1 Scanning electron microscopy images of (A) PCTs and (B) PCRs. The walls of the PCTs had a thickness of $\sim 20 \pm 5 \mathrm{~nm}(\sim 40 \pm 10 \mathrm{~nm}$ at the pore mouths of the AAO pores). The scale bars correspond to $1 \mu \mathrm{m}$. substrate and $k$ is the spring constant of the AFM cantilever. Measurements on 13 different PCTs revealed an average Young's modulus of $22 \pm 10 \mathrm{GPa}$. Strikingly, this value is one order of magnitude higher than that of bulk polycyanurate (3.46 GPa). ${ }^{1}$

According to the polymer-fiber theory, the mechanical strength of polymer fibers increases with orientation of the polymer molecules. ${ }^{36}$ Thus, the drastic increase in Young's modulus of the PCTs might be indicative of ordering on a molecular scale.

Molecular ordering is associated with optical anisotropy leading to birefringence. Indeed, as revealed by POM, both PCTs and PCRs are birefringent. About $150 \mu \mathrm{m}$ thick bundles of aligned PCTs lying flat on clean glass slides located on a POM stage between crossed polarizers showed identical dependence of the birefringence $\Delta n$ on the rotation angle $\alpha$ that was varied by rotating the sample stage. While polarizer and analyzer remained fixed, the relative orientation of the PCT/PCR long axes to the transmission planes of polarizer and analyzer was altered by rotating the sample stage. Thus, $\alpha$ is the angle between the transmission plane of the polarizer and the PCT/ PCR long axes, while $90^{\circ}-\alpha$ corresponds to the angle between the transmission plane of the analyzer and the long axes of the PCTs and PCRs. At $\alpha=0^{\circ}$ (Fig. 3A(a)), the long axes of the PCTs were aligned with the transmission plane of the polarizer and oriented perpendicular to the transmission plane of the analyzer. While the PCTs appear dark at $\alpha=0^{\circ}$, their brightness increased when $\alpha$ was increased from $0^{\circ}$ to $15^{\circ}$ to $30^{\circ}$, reached a maximum at $\sim 45^{\circ}$ (Fig. 3A(b)) and decreased again when $\alpha$ was further increased from $45^{\circ}$ to $60^{\circ}$ to $75^{\circ}$. At $\alpha=90^{\circ}$, the PCTs appeared again dark (Fig. 3A(c)). The integrated brightness (the sum of the brightness values of all individual pixels) of the micrographs shown in Fig. $3 \mathrm{~A}$ is indicative of the relative intensity of the transmitted light $I_{\text {trans }}$. The dependence of $I_{\text {trans }}$ on $\alpha$ (Fig. 3B) can be fitted with a sinusoidal curve having maximum values at $\alpha=45^{\circ}$ and $\alpha$ values which are multiples of $45^{\circ}$. Minima of $I_{\text {trans }}$ occur at $\alpha=0^{\circ}, \alpha=90^{\circ}$ and $\alpha=180^{\circ}$, respectively. Hence, maximum light transmission was observed if the long axes of the PCTs were inclined by $45^{\circ}$ with respect to the transmission planes of polarizer and analyzer. The observed periodic $I_{\text {trans }}(\alpha)$ profiles for PCTs and PCRs (Fig. S2 $\dagger$ ) resemble the $I_{\text {trans }}(\alpha)$ profiles resulting from planar alignment of the thermotropic or lyotropic liquid-crystalline species. ${ }^{37}$ The transmitted light intensity $T$ and the birefringence $\Delta n$ are related as follows (see Methods in ESI $\dagger$ ):

$$
T=\frac{I_{\text {trans }}}{I_{0}}=\sin ^{2}\left(\pi \frac{d}{\lambda} \Delta n\right)
$$

$I_{0}$ is the incident light intensity, $d$ the bundle thickness $(\sim 150 \mu \mathrm{m})$, and $\lambda$ the wavelength of the incident light $(\lambda=850 \mathrm{~nm})$. Accordingly, the average $\Delta n$ at the angle of $45^{\circ}$ is calculated from eqn (3) to be $0.0123 \pm 0.0002$. This value is in reasonable agreement with that determined from the second order of retardation according to the Michel-Levy chart at $45^{\circ}$ in digitally captured polarization color images $(\Delta n=0.02)$ (Fig. 3A).

For comparison, we also studied bulk CEM and PCN samples by POM. Liquid CEM was deposited on a glass slide and covered 

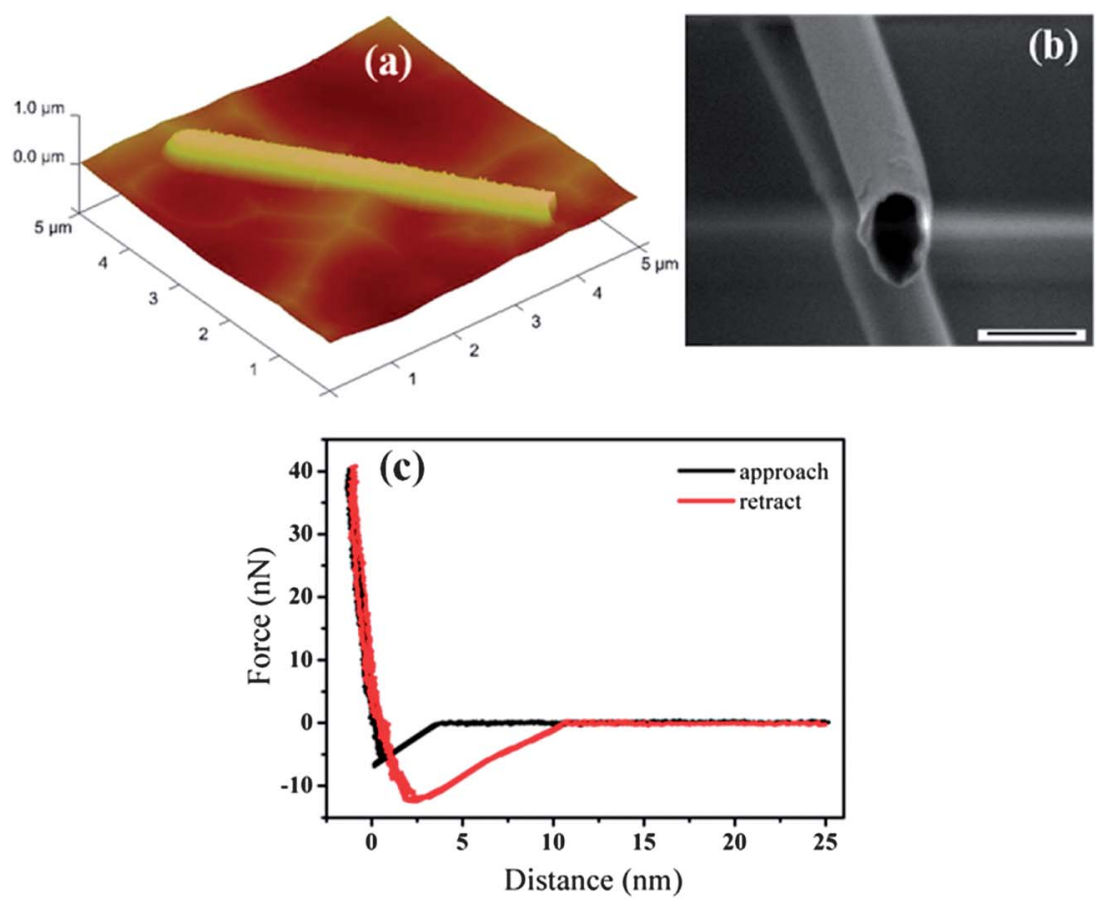

Fig. 2 (A) AFM image of a single PCT. (B) SEM image of a single PCT. (C) Force-distance curves recorded on a single PCT (diameter $\sim 380 \mathrm{~nm}$; wall thickness $\sim 20 \mathrm{~nm}$ ) during approach and retraction of the cantilever.

by a second glass slide. To ensure defined and homogeneous CEM film thickness $(\sim 150 \mu \mathrm{m})$ polystyrene beads were used as spacers. A $150 \mu \mathrm{m}$ thick bulk PCN film was prepared on a glass slide using the same curing program (see Experimental section) as for PCRs and PCTs. Neither bulk CEM films nor bulk PCN films probed in the same way as PCTs and PCRs showed even a weak indication of birefringence (Fig. S3†).

DSC curves of bulk CEM as well as of CEM nanotubes inside AAO obtained by infiltration at $80{ }^{\circ} \mathrm{C}$ and CEM nanorods inside AAO obtained by infiltration at $120^{\circ} \mathrm{C}$ are shown in Fig. S4. $\dagger$ Bulk CEM has a $T_{\mathrm{g}}$ of about $-14.6^{\circ} \mathrm{C}$, while $T_{\mathrm{g}}$ of CEM nanotubes amounted to $1.20^{\circ} \mathrm{C}$ and that of CEM nanorods to $4.9{ }^{\circ} \mathrm{C}$. As obvious from the DSC measurements, thermal curing of bulk CEM is an exothermic process that sets in at $\sim 150{ }^{\circ} \mathrm{C}$, while the curing peak shows a maximum at $\sim 250{ }^{\circ} \mathrm{C}$. However, in the case of CEM confined to AAO curing already started at $\sim 120^{\circ} \mathrm{C}$ (CEM nanotubes in AAO) and $150{ }^{\circ} \mathrm{C}$ (CEM nanorods in AAO). The curing peaks in the DSC curves had maxima of $205{ }^{\circ} \mathrm{C}$ (CEM nanotubes in AAO) and $224^{\circ} \mathrm{C}$ (CEM nanorods in AAO). Thus, curing of CEM in AAO occurred at temperatures about $40 \mathrm{~K}$ below the temperatures at which curing of bulk CEM took place. This outcome indicates higher reactivity of CEM located in AAO. Fig. 4 shows DSC traces of bulk PCN as well as of released PCRs and PCTs $380 \mathrm{~nm}$ in diameter. The DSC trace of bulk PCN shows a glass transition at $T_{\mathrm{g}} \sim 238^{\circ} \mathrm{C}$ but no melting peaks. Strikingly, PCRs showed two broad melting peaks at $\sim 95{ }^{\circ} \mathrm{C}$ and $\sim 249{ }^{\circ} \mathrm{C}$, but no apparent glass transition. PCTs showed only a single, relatively narrow melting peak at $\sim 144{ }^{\circ} \mathrm{C}$.

Ordering and orientation effects in CEM nanorods, CEM nanotubes, PCTs and PCNs located in the aligned nanopores of AAO were studied by $\theta / 2 \theta$ scans and by Schulz scans (note that the CEM nanotubes were located in AAO pores with a diameter of $180 \mathrm{~nm}$, whereas for all other samples AAO with a pore diameter of $380 \mathrm{~nm}$ was used). In the $\theta / 2 \theta$ scans of CEM nanorods, CEM nanotubes, PCRs and PCTs (Fig. 5A) broad reflections at $2 \theta \approx 8.5^{\circ}(d \approx 1.03 \mathrm{~nm})$ and $2 \theta \approx 21^{\circ}$ $(d \approx 0.42 \mathrm{~nm}$ ) appeared, while the $\theta / 2 \theta$ pattern of bulk PCN films with a thickness of $\sim 150 \mu \mathrm{m}$ exclusively contained an amorphous halo between $2 \theta \approx 15^{\circ}$ and $2 \theta \approx 30^{\circ}$ (Fig. S5 $\dagger$ ). Schulz scans for the reflection at $2 \theta \approx 8.65^{\circ}$ measured for PCRs, PCTs and CEM nanorods showed a peak at $\Psi=0^{\circ}$ (Fig. 5B).

The X-ray measurements suggest that the interplay of a hard AAO surface and cylindrical confinement induces anisotropic ordering of CEM inside the AAO pores, which is conserved upon curing. Thus, PCTs and PCNs show melting peaks in DSC heating scans, anisotropic birefringence and significantly enhanced interfacial stiffness. However, the observed interfaceinduced anisotropic ordering does not result in crystalline longrange ordering. The features apparent in the X-ray patterns and the DSC scans rather suggest the occurrence of pseudo-nematic short-range ordering, which nevertheless significantly influences the structure and properties of PCNs at hard surfaces and may even dominate the behaviour of PCN nanocomposites with high volume fractions of interphases.

The question arises as to why CEM molecules adopt a preferred orientation at the AAO pore walls. It is certainly straightforward to assume that the concave pore walls guide the arrangement of the CEM molecules. However, it is also interesting to note that interface-induced anisotropic ordering of CEM in AAO is accompanied by enhanced reactivity. As obvious 
(A)

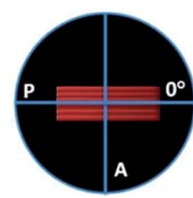

(a)

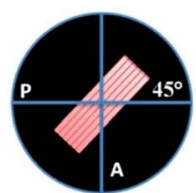

(b)

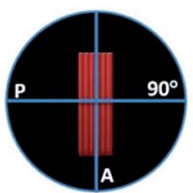

(c)

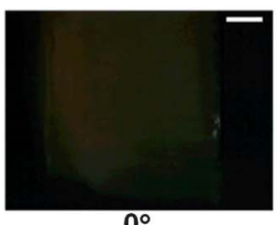

$0^{\circ}$

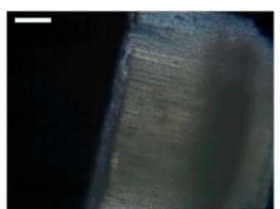

$15^{\circ}$

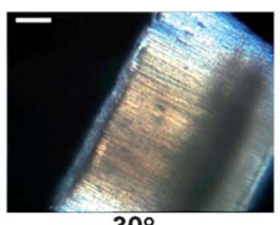

$30^{\circ}$

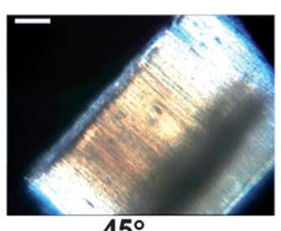

$45^{\circ}$

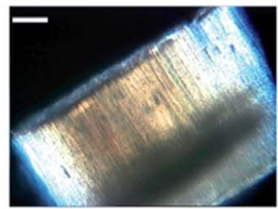

$60^{\circ}$

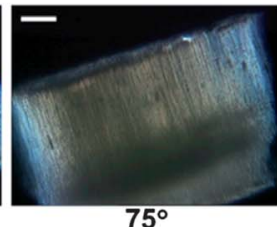

$75^{\circ}$

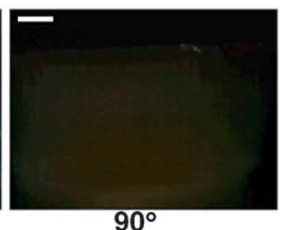

(B)

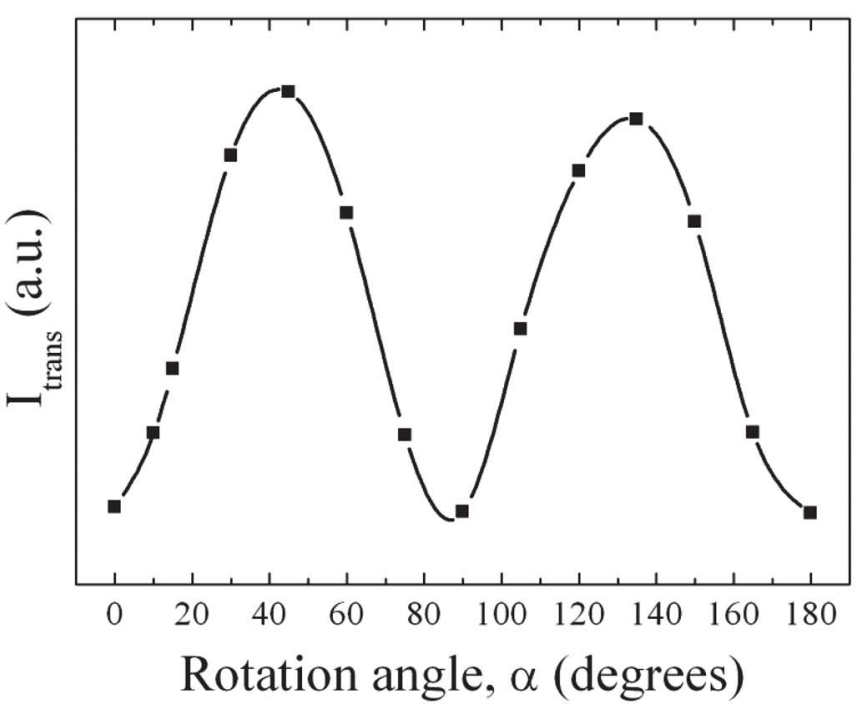

Fig. 3 (A) POM of aligned PCTs with a diameter of $380 \mathrm{~nm}$ lying planar on a glass slide at rotation angles $\alpha$ of $0^{\circ}, 15^{\circ}, 30^{\circ}, 45^{\circ}, 60^{\circ}, 75^{\circ}$ and $90^{\circ}$. The scale bars correspond to $20 \mu \mathrm{m}$. (B) Integrated transmitted light intensity I trans of PCTs as a function of $\alpha$. The solid curve is a guide to the eye.

from Fig. S4, $\dagger$ curing of CEM located in AAO starts at lower temperatures than curing of bulk CEM. We speculate that the AAO pore walls catalyze the curing reaction. If so, specific interactions between CEM and AAO pore walls responsible for the pore walls' catalytic activity may also play a role in the generation of anisotropic ordering. Vice versa, geometryinduced anisotropic ordering may facilitate catalysis of the curing reaction.

\section{Conclusions}

Using self-ordered AAO containing arrays of aligned cylindrical nanopores as inorganic model matrices, we have shown that precursors of polycyanurate thermosets show interface-induced anisotropic near ordering characterized by uniform uniaxial orientation with respect to the AAO pore axes. The anisotropic interface-induced near-ordering is conserved upon curing, as evidenced by polarizing microscopy, thermal analysis and X-ray scattering. The uniaxial anisotropy characterizing interfaceinduced ordering of polycyanurate thermosets inside AAO could be evidenced by X-ray texture analysis; its conservation in released one-dimensional polycyanurate thermoset nanostructures could be evidenced by the pronounced periodical change in birefringence during rotation between crossed polarizers. The anisotropic surface-induced ordering in onedimensional polycyanurate thermoset nanostructures results in a Young's modulus one order of magnitude higher than that reported for bulk polycyanurate, as revealed by AFM. 


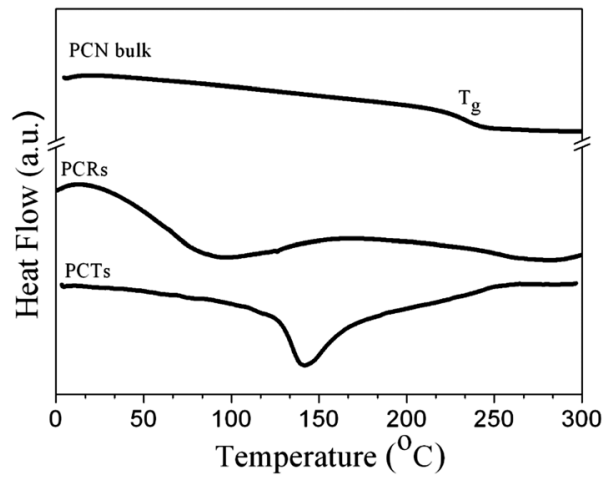

Fig. 4 DSC heating runs (heating rate $10 \mathrm{~K} \mathrm{~min}^{-1}$ ) of bulk PCN, of released PCRs with a diameter of $380 \mathrm{~nm}$ and of released PCTs with a diameter of $380 \mathrm{~nm}$ and a wall thickness of $20 \mathrm{~nm}$

(A)

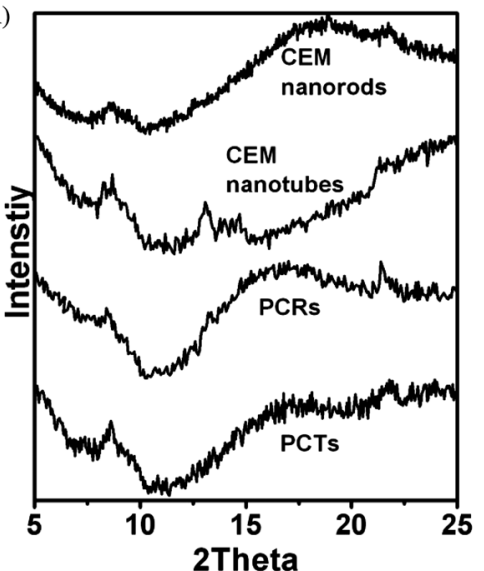

(B)

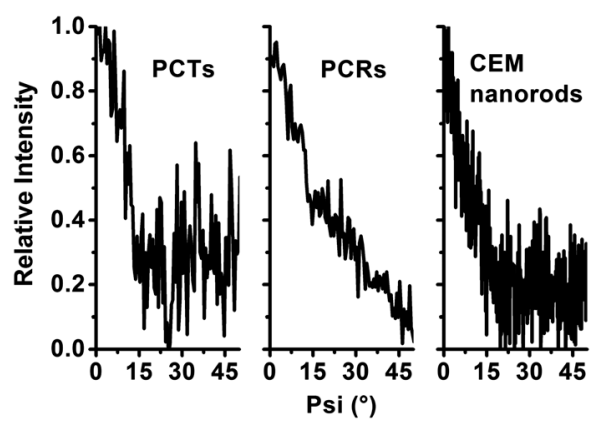

Fig. 5 (A) $\theta / 2 \theta$ scans of CEM nanorods, CEM nanotubes, PCRs and PCTs located in AAO. Note the pore diameter of the AAO membrane containing CEM nanotubes was $180 \mathrm{~nm}$, while all other AAO membranes used had a pore diameter of $380 \mathrm{~nm}$. (B) Schulz scans of PCTs, PCRs and CEM nanorods confined to AAO with a pore diameter of $380 \mathrm{~nm}$ for $2 \theta=8.65^{\circ}$.

Polycyanurate thermosets are a technologically important class of materials. During moulding processes the polycyanurate thermosets are inevitably in contact with the hard surfaces of the moulds. Moreover, polycyanurate thermosets blended with inorganic filler particles have high specific interfacial area with the hard surfaces of the filler particles. The results presented here shed light on the structure formation of polycyanurate thermosets at hard surfaces. The findings reported here may improve the understanding of polymer/solid interfaces, as required for the design of advanced nanocomposites for applications in aviation and high-speed electronics, and for the design of mechanical hybrid nanostructures for advanced biomimetic adhesive systems.

\section{Acknowledgements}

The authors thank C. Hess and H. Tobergte for the preparation of AAO membranes, A. Best for the POM measurements, G. Glasser for SEM images and K. Sklarek (MPI Halle) for additional SEM investigations. H.D. and M.S. gratefully acknowledge financial support from the German Research Foundation (SPP 1369, STE 1127/13, INST 190/134-1 FUGG). B.Y. acknowledges financial support from HEC, Pakistan.

\section{Notes and references}

1 B. Yameen, H. Duran, A. Best, U. Jonas, M. Steinhart and W. Knoll, Macromol. Chem. Phys., 2008, 209, 1673.

2 Advances in Polymer Science, Springer Tracts in New Polymerisation Techniques and Synthetic Methodologies Series, Vol. 155, ed. P. Hodge, Springer-Verlag, BerlinHeidelberg, 2001.

3 V. P. McConnell, Resins for the Hot Zone Part II: BMIs, CEs, Benzoxazines \& Phthalonitriles, High Perform. Compos., 2009, 43-49.

4 F. Chao, N. Bowler, X. L. Tan, G. Z. Liang and M. R. Kessler, Composites, Part A, 2009, 40, 1266.

5 G. G. Barclay, C. K. Ober, K. I. Papathomas and D. W. Wang, Macromolecules, 1992, 25, 2947.

6 A. J. Guenthner, K. R. Lamison, V. Vij, J. T. Reams, G. R. Yandek and J. M. Mabry, Macromolecules, 2012, 45, 211.

7 R. J. Zaldivar, Lessons Learned in the Processing of Polycyanurate Resin Composites, The Aerospace Corporation Laboratory Operations Report TR-98(8565)-8, El Segundo, 2002.

8 S. B. He, G. Z. Liang, H. X. Yan, J. H. Wang and L. L. Yang, Polym. Adv. Technol., 2009, 20, 143.

9 J. N. Hay, Processing and Cure Schedules for Cyanate Ester Resins, ed. I. Hamerton, Chemistry and Technology of Cyanate Esters, Blackie Academic and Professional, Glasgow, 1994, ch. 6, p. 178.

10 M. Steinhart, S. Zimmermann, P. Göring, A. K. Schaper, U. Gösele, C. Weder and J. H. Wendorff, Nano Lett., 2005, 5, 429.

11 M. Steinhart, P. Göring, H. Dernaika, M. Prabhukaran, U. Gösele, E. Hempel and T. Thurn-Albrecht, Phys. Rev. Lett., 2006, 97, 027801.

12 S. Gruener and P. Huber, Phys. Rev. Lett., 2009, 103, 174501.

13 S. V. Primak, T. Jin, A. C. Dagger, D. Finotello and E. K. Mann, Phys. Rev. E: Stat., Nonlinear, Soft Matter Phys., 2002, 65, 031804.

14 P. J. Sebastião, D. Sousa, A. C. Ribeiro, M. Vilfan, G. Lahajnar, J. Seliger and S. Žumer, Phys. Rev. E: Stat., Nonlinear, Soft Matter Phys., 2005, 72, 061702. 
15 S. G. Cloutier, J. N. Eakin, R. S. Guico, M. E. Sousa, G. P. Crawford and J. M. Xu, Phys. Rev. E: Stat., Nonlinear, Soft Matter Phys., 2006, 73, 051703.

16 F. Grinberg, Magn. Reson. Imaging, 2007, 25, 485.

17 A. V. Kityk, M. Wolff, K. Knorr, D. Morineau, R. Lefort and P. Huber, Phys. Rev. Lett., 2008, 101, 187801.

18 A. V. Kityk and P. Huber, Appl. Phys. Lett., 2010, 97, 153124. 19 Y. Bai and N. L. Abbott, Langmuir, 2011, 27, 5719.

20 Q. Ji, R. Lefort, R. Busselez and D. Morineau, J. Chem. Phys., 2009, 130, 234501.

21 B. M. Ocko, H. Hlaing, P. N. Jepsen, S. Kewalramani, A. Tkachenko, D. Pontoni, H. Reichert and M. Deutsch, Phys. Rev. Lett., 2011, 106, 137801.

22 H. Masuda, K. Yada and A. Osaka, Jpn. J. Appl. Phys., 1998, 37, L1340.

23 J. L. Hutter and J. Bechhoefer, Rev. Sci. Instrum., 1993, 64, 1868.

24 H.-J. Butt, B. Capella and M. Kappl, Surf. Sci. Rep., 2005, 59, 1. 25 P. J. Pablo, I. A. T. Schaap, F. C. MacKintosh and C. F. Schmidt, Phys. Rev. Lett., 2003, 91, 098101.

26 L. G. Schulz, J. Appl. Phys., 1949, 20, 1030.
27 W. P. Chernock and P. A. Beck, J. Appl. Phys., 1952, 23, 341. 28 H. W. Fox, E. F. Hare and W. A. Zisman, J. Phys. Chem., 1955, 59, 1097.

29 M. Zhang, P. Dobriyal, J.-T. Chen, T. P. Russell, J. Olmo and A. Merry, Nano Lett., 2006, 6, 1075.

30 H. Xiang, K. Shin, T. Kim, S. I. Moon, T. J. McCarthy and T. P. Russell, Macromolecules, 2004, 37, 5660.

31 M. Steinhart, J. H. Wendorff, A. Greiner, R. B. Wehrspohn, K. Nielsch, J. Schilling, J. Choi and U. Gösele, Science, 2002, 296, 1997.

32 B. Pulamagatta, M. Y. E. Yau, I. Gunkel, T. Thurn-Albrecht, K. Schröter, D. Pfefferkorn, J. Kressler, M. Steinhart and W. H. Binder, Adv. Mater., 2011, 23, 781.

33 N. Shingne, M. Geuss, B. Hartmann-Azanza, M. Steinhart and T. Thurn-Albrecht, Polymer, 2013, 54, 2737.

34 R. Lenormand, J. Phys.: Condens. Matter, 1990, 2(suppl. A), 79.

35 M. G. Bernadiner, Transp. Porous Media, 1998, 30, 251.

36 T. Liu and S. Kumar, Nano Lett., 2003, 3, 647.

37 P. J. Collings and M. Hird, Introduction to Liquid Crystals, Taylor \& Francis, London, 1997. 